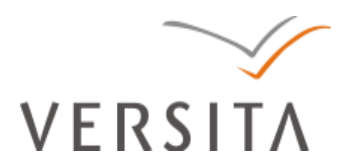

Folia Oeconomica Stetinensia

DOI: 10.2478/v10031-011-0045-7

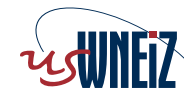

Wydzial Nauk Ekonomicznych i Zarządzania Uniwersytetu Szczecíískiego

\title{
DILEMMAS OF ECONOMIC MEASUREMENTS IN WEAK SCALES
}

Jerzy W. Wiśniewski, Prof.

Nicolaus Copernicus University

Department of Econometrics and Statistics

Faculty of Economics and Management

Gagarina 13a, 87-100 Toruń

e-mail: jerzy.wisniewski@umk.pl

Received 30 September 2011, Accepted 19 January 2012

\begin{abstract}
The attempts to measure economic phenomena and processes of qualitative nature have become commonplace among scholars engaged in empirical research. The use of measurement in the nominal and ordinal scales, however, requires a thorough knowledge of the meaning of numbers generated in the course of such operations. It is also necessary to have knowledge of acceptable statistical and econometric tools to be used in such research. Unfortunately, researchers often apply methods that are not acceptable for the numbers in the weak scales. As a result they "soup up" the outcome of their empirical research, instead of achieving a substantive truth. This article is devoted to the possibilities of statistical analysis of numbers resulting from the weak scale measurement.
\end{abstract}

Keywords: weak scales, statistical analyses of qualitative variables.

JEL classification: $\mathrm{C} 25, \mathrm{C} 81$. 


\section{Introduction}

In the economic science it is quite common to measure the quality characteristics by numbers belonging to the so-called weak scale. Their use requires in such cases appropriate statistical tools. The application of statistical methods that are not adequate to the scale of numbers belonging to poor results in cognitive errors which lead to absurd interpretation of test results.

\section{Measuring scales}

For the last quarter of a century an awareness of the existence of four measuring scales has been developing in the environment of economists ${ }^{1}$. As listed according to the power of numbers, from the weakest to the strongest, the following scales are distinguished ${ }^{2}$ :

- nominal,

- ordinal,

- interval,

- ratio (absolute).

The nominal and ordinal scales belong to weak categories, while the remaining two form a group of strong scales. Weak scales are used mainly for the measurement of phenomena and processes of a quality character (descriptive). They can also be used for transforming results of the measurement in strong scales in order to eliminate the unnecessary information overload.

\section{The measurement in weak scales}

Numbers which we deal with in present times most often belong to the results of measurement in the nominal scale. They play the role of ID badges that enable us to distinguish various objects or their traits. The man is described with such numbers already at the moment of the birth. The first one is the PESEL (the Common Electronic System of Population Register) associated with the date of birth. Becoming an adult a citizen is given the NIP (a Taxpayer Identification Number) granted by tax services. At school it is the number of a student card, at the university - the number of the student's credit book. Then, there is a phone number, etc.

Numbers in the nominal scale are used for marking, identification or classification of separate categories ${ }^{3}$. The allotted numbers usually play here a role of symbols that replace names or verbal descriptions. In this scale the only acceptable relations among numbers are: a) the equality of elements within particular categories, e.g. $a=b$, or b) the dissimilarity of 
separate categories, e.g. $b \neq c$. The only acceptable arithmetical procedure is summing up, the result of which is generally a natural number. As far as statistical techniques are concerned only the ones which rely on counting are allowed.

The nominal scale has an element worth noting - a dichotomous scale. It is frequently used in statistical surveys. This scale is used to distinguish pairs of separate categories. Simultaneous defining of the variant $A$ of the observed phenomenon permits classifying the events in the form

of variants: A or $\hat{A}$ (not $A)$. Assigning number 1 to each observation $A$, and number 0 to the observation $\hat{A}$ creates the so-called dummy variable.

In the ordinal scale ranks the numbers are ranks denominating the order of elements or characteristics of a phenomenon. Ranks reflect not only the elements or inequality, but also their arrangement in terms of the present ownership. Categories considered here are separable phenomena. The figures in this scale are comparable because of the module. But they have only a relative (not absolute) importance since the distance between ranks is not known. Furthermore, the distances between adjacent ranks are unequal. Thereby it is possible to compare the ranks by determining both the relationship of equality and the majority, and consequently it is possible to define the minority, such as $a>b>c>\ldots>z$. It is not possible to determine the distance between ranks or to decide if they differ.

Note the possibility of objective and subjective measurement. The existence of a pattern, which compares the measured object or feature, allows obtaining an objective measurement results. We observe such cases when we take measurements allowing to obtain a result expressed in physical units, such as weight, length, volume, or a value in monetary units. The lack of the precisely defined standard results in the measurement of a subjective character. All measurements of features based on asking respondents to put them in the order of e.g. importance resulting in the form of ranks, belong to subjective categories.

\section{Arithmetic operations on numbers in a variety of scales}

All arithmetic operations (addition, subtraction, multiplication, division) are permitted on a series of numbers that have the following characteristics:

a) natural zero is known for a given feature,

b) distances between numbers are known,

c) distances between adjacent numbers are measured in units and are identical for each adjacent pair. 
All these properties are demonstrated only by the numbers that belong to the results of the ratio (absolute) measurement. Especially the division requires from a series to have each of the above properties. If we do not know the natural zero in a series we cannot determine the proportion of pairs of numbers. For example, the results of measuring the temperature on the Celsius scale do not allow for the comparison of two temperatures in the form of a quotient. If on a specific day (and in a specific place) the temperature at $12: 00$ was $6^{\circ} \mathrm{C}$, and on the previous day at the same time - only $3^{\circ} \mathrm{C}$, we cannot say that on the former day the temperature was twice higher than on the previous day. One can only conclude that the temperature that day was higher by $3^{\circ} \mathrm{C}$ in comparison to the previous day. The measurement result belongs to the interval scale 4 , in which there natural zero is not known.

The operations of addition and subtraction require fulfilling the conditions $b$ and $c$, i.e. equal and the distance in units between adjacent numbers. Imagine the sequence of digits: 566114602 which, firstly, means the net proceeds from the sale of a joint stock company (in PLN); secondly, is a number assigned to an adult citizen of China according to height ranking; and thirdly, is a phone number in the Department of Econometrics and Statistics at Nicolaus Copernicus University. The same set of numbers, but what a different meaning of each of these numbers and what a variety of analytical capabilities. The revenue belongs to the proportional scale, which allows the use of any arithmetic operation on the set of such numbers. The number assigned according to height should be measured on the ordinal scale and means just that 566114601 citizens in China are taller (or not shorter) than that specific person. No arithmetic operations on such numbers are allowed. The phone number is only an identifier that makes it possible to contact the person to whom it is assigned; this number belongs to the nominal measurement results.

Let us measure the ordinal scale results. A classic example of the objective existence of the so-called ranks are uniformed services (army, police, firefighters, etc.). Let us try to analyze the sense of the military rank accumulation operations. As a principle a colonel has to wait a long time for their promotion to the general's position, and only some of the officers of this rank become generals. Suppose that the operation of summing military ranks is allowed. In such a case the father in the rank of a colonel could send his son to a non-commissioned officer school so that his son can obtain the corporal's rank. After obtaining the rank of corporal the son waivers his rank for the favour of his father who can add the rank of corporal to obtain a general's degree. Is this logical? Only seemingly. Such an operation is impossible because ranks are not additive. Note the different distance between the various military degrees of military. The distance between brigadier general is much greater than the distance between 
second lieutenant and lieutenant, although in both cases we deal with the neighboring ranks. Summing up the ranks can lead to absurd results. This means that determining the arithmetic average for a number of ranks is unacceptable.

Also ranks subtraction can lead to absurd findings. We can only determine the number of levels to be reached to be granted a desired rank. For example, to achieve a rank of a brigadier general while being a captain, you must pass four consecutive ranks: of a major, a lieutenant colonel and a colonel, and finally a general. What is more, the higher the rank, the harder it is to get. There is no linearity here, allowing for any addition or subtraction of ranks. But the fundamental issue is the lack of knowledge about the distances between the ranks, which prevents any arithmetic operations.

\section{Statistical tools allowed in the weak scales}

In many scientific works we find addition and subtraction of ranks, setting the arithmetic mean, variance, etc., while as far as the ranks are concerned we can use only a range of statistical tools, based on position measures. Thus we are allowed to apply instruments linked to fractionation, including the relevant statistical tests. In the rank measurement, however, it is not possible to make the direct use of the tools of the analysis of correlation and regression due to the inadmissibility of arithmetic operations on numbers of this class.

The so-called Spearman "coefficient of rank correlation" commonly used in such situations has been derived from the Pearson correlation coefficient to be applied for the sequences of one pair of natural numbers of $\mathrm{n}$ observations. Pearson's correlation coefficient takes the following form:

$$
\rho=\frac{\operatorname{cov}\left(x_{i}, x_{j}\right)}{\sqrt{\operatorname{var}\left(x_{i}\right) \operatorname{var}\left(x_{j}\right)}}
$$

for any pair of variables $X_{i}$ and $X_{j}$ it takes the form:

$$
r_{i j}=\frac{\sum_{t=1}^{n}\left(x_{i t}-\bar{x}_{i}\right)\left(x_{j t}-\bar{x}_{j}\right)}{\sqrt{\sum_{t=1}^{n}\left(x_{i t}-\bar{x}_{i}\right)^{2}\left(x_{j t}-\bar{x}_{j}\right)^{2}}}
$$

If the observations on the variables $X_{i}$ and $X_{j}$ are natural numbers ${ }^{5}$, i.e., $x_{i t}=1, \ldots, n, x_{j t}=1, \ldots, n$ $(t=1, \ldots, n)$, then the correlation coefficient (2) develops into the Spearman coefficient: 


$$
r_{s}=1-\frac{6 \sum_{t=1}^{n} d_{t}^{2}}{n\left(n^{2}-1\right)}
$$

which can be easily proved. Factor (3) can therefore be used when the observations on each pair of variables are natural numbers belonging to relative measurement results.

So there is a question whether in case of the rank measurement the researcher is helpless when facing questions about the interdependence or the correlation of features? It can be an effective solution to recast the ranks into dummy variables. This allows for the analysis of the association of attributes (associations, contra associations). Suppose that we study the behavior of consumers who are expected to put some product features in the order of importance. The obtained results for a particular feature (e.g., durability) can be associated with the respondents' education ${ }^{6}$. The conversion of ranks in a dummy variable in such a way that the number 1 is assigned to the observations of the variable $X_{j}$ for which the respondent pointed ${ }^{7}$ the rank of 1 or 2 , i.e.:

$$
x_{t j}= \begin{cases}1, & \text { when the rank equals } 1 \text { or } 2, \\ 0, & \text { in other cases }\end{cases}
$$

where $t$ is the number of statistical observation (respondent), $(t=1, \ldots, n)$.

The study of the association of a customer's preference of durability with their education requires distinguishing a certain education level (type) by means of another dummy variable $X_{i}$, marking the type A education (e.g. university degree, at least bachelor's degree), i.e.:

$$
x_{t i}= \begin{cases}1, & \text { for the respondents on A level, } \\ 0, & \text { in other cases. }\end{cases}
$$

In such a case we can use the following attributes association coefficient (4), using the

\begin{tabular}{|c|c|c|c|c|}
\hline \multirow{2}{*}{\multicolumn{2}{|c|}{ Dummy variable }} & \multicolumn{2}{|c|}{$X_{j}$} & \multirow{3}{*}{$\begin{array}{c}\text { Sum } \\
n_{00}+n_{01}=q_{i}\end{array}$} \\
\hline & & \multirow{2}{*}{$\begin{array}{c}x_{t j}=0 \\
n_{00}\end{array}$} & \multirow{2}{*}{$\begin{array}{c}x_{t j}=1 \\
n_{01}\end{array}$} & \\
\hline$X$ & $x_{t i}=0$ & & & \\
\hline$\Lambda_{i}$ & $x_{t i}=1$ & $n_{10}$ & $n_{11}$ & $n_{10}+n_{11}=p_{i}$ \\
\hline \multicolumn{2}{|c|}{ Sum } & $n_{00}+n_{10}=q_{j}$ & $n_{01}+n_{01}=p_{j}$ & $n=q_{i}+p_{i}=p_{j}+q_{j}$ \\
\hline
\end{tabular}
following bipartite table.

Table 1 . The aggregate numbers of observations of the pair of dummy variables $X_{i}$ and $X_{j}$

Source: Wiśniewski (1986), pp. 59-60. 


$$
r_{i j}=\frac{A+B-(C+D)}{n \sqrt{\left(n_{11}+n_{10}\right)\left(n_{00}+n_{01}\right)\left(n_{00}+n_{10}\right)\left(n_{11}+n_{01}\right)}}
$$

where:

$$
\begin{aligned}
& A=n_{00}\left(n_{11}+n_{10}\right)\left(n_{11}+n_{01}\right), \\
& B=n_{11}\left(n_{00}+n_{01}\right)\left(n_{00}+n_{10}\right), \\
& C=n_{10}\left(n_{00}+n_{01}\right)\left(n_{11}+n_{01}\right), \\
& D=n_{10}\left(n_{11}+n_{01}\right)\left(n_{00}+n_{10}\right) .
\end{aligned}
$$

The coefficient of association (4) has been transformed from the Pearson correlation coefficient for the sake of the frequency analysis ${ }^{8}$. It reaches classical values, i.e. $-1 \leq r_{i j} \leq 1$. It is also possible to test the relevance of this association factor.

The conversion of the ordinal measurement results into dummy variables ${ }^{9}$ increases the possibility of applying the tools of statistics and econometrics in comparison with the potential of the ordinal scale. Measurement results in the form of dummy variables also allow for the use of regression models, especially for aggregates ${ }^{10}$. Therefore, it is worthwhile to compare the benefits of increasing the analytical capacity of the nominal scale, measured by dummy variables, against the loss of information in the ranks.

\section{Alternative proposals}

Adequate planning of the empirical experiment - in the case of surveys - helps to avoid difficulties arising from the results of surveying respondents about range arrangement of features or objects. It is recommended to replace range with relatively precisely defined points ${ }^{11}$. So we can allocate, for example, 100 points to a respondent, which in turn they can distribute with an accuracy of 0.5 points or more. This allows us to obtain the numbers of known and unit distance between them, making them adherent to the relative scale. However, we still remain in the area of subjective measurement, which cannot be avoided ${ }^{12}$.

An alternative solution could be to attribute points to each feature (object) such as a number between 1 to 10 , with an accuracy of 0.5 points, while the more important the feature, the more points it receives. The measurement is still subjective, but we again know the distances between the numbers and they are units.

Replacing ranks with the measurement outcomes in a form of points eliminates the biggest disadvantage of ranks, which is the inability to use any arithmetic operations. Points assigned by a respondent are the numbers belonging to the ratio scale and their nature is stochastic 
and subjective at the same time. Therefore it is acceptable in this case to apply all analytical methods that are adequate to the relative scale. Because of the subjectivity of the measurement and its stochastic nature it is necessary, however, to be slightly more careful when drawing statistical conclusions than in the case of analyzing measurement results obtained in the form of conventional physical units (kilograms, meters, seconds, etc.).

These solutions enhance our capacity to use the tools of econometrics and statistics in processing measurement results. It should be noted that the obtained results are eligible for a class of limited variables that play in the model a role of explanatory variables of the observations $y_{t}^{(o)}$ that can have even bilateral restrictions.

Their specificity is to have lower and upper limits ${ }^{13}$ :

$$
y_{\min } \leq y_{t}^{(o)} \leq y_{\max }
$$

where $y_{\min }$ is the lowest possible value of the observation of the considered variable, while $y_{\max }$ is the highest possible value of observation of this response variable.

A possible solution may then be to apply one of many possible transformations of a limited dependent variable. The first is the basic transformation of the limited variable, given by:

$$
y_{t}^{(p)}=\frac{y_{t}^{(o)}-y_{\min }}{y_{\max }-y_{t}^{(o)}}
$$

where the signs are identical, as in the formula (5). And $y_{t}^{(p)}$ is a basic transformation of the bilaterally limited variable $y_{t}^{(o)}$. The basic transformation of a limited dependent variable converts it into a variable that takes values from the interval $0 \leq y_{t}^{(p)} \leq \infty$.

A variable in a form of $y_{t}^{(p)}$ is unlimited in non-negative values. However, it still has a bottom limit on the level of 0 ; so it has characteristics of many economic variables, which achieve non-negative values ${ }^{14}$.

Another important variable transformation of bilaterally limited variable is the logit transformation, which is given by:

$$
y_{t}^{(l)}=\ln y_{t}^{(p)}=\ln \frac{y_{t}^{(o)}-y_{\min }}{y_{\max }-y_{t}^{(o)}}
$$

So the logit transformation of the limited variable is the logarithm of the basic transformation. It converts a bilaterally limited variable to an unlimited variable. Therefore it should be noted that the variable in a logit form satisfies the inequality: $-\infty \leq y_{t}^{(l)} \leq+\infty$. 


\section{Conclusions}

Expert use of analytical tools in relation to the accumulated statistical material is a prerequisite for the correct description of reality and its reliable cognition. Meeting the principles outlined in this paper can prevent researchers from errors, leading to the knowledge of the erroneous or fictitious character. Mistakes in the use of statistics and econometrics instruments can lead to irrational decisions when managing companies or tasks. It is less risky when false results of a cognitive process land on bookshelves where they are left forgotten.

\section{Notes}

1 A metrology, which is a field of science, knowledge and technology, deals with everything that is connected with measurements. „All phases of measurement are the object of the metrology: establishing the model of the measured object and what is being measured up, designing and preparing the measuring system, performing the measurement itself and processing the measurement results, including determining parameters that describe the inaccuracy of measurement. Metrology also cover the establishment of the units of measures" comp. Encyclopedia (2004), p. 628.

2 The author of theory of measuring scales is S.S. Stevens. Stevens (1946).

3 These issues were widely discussed in the book by Wiśniewski (1986), the first chapter.

4 In statistics and econometrics sometimes operations are conducted of normalizing or the standardizing a random variable. As a result, a "new" zero in the statistical series appears, not being a natural nil. In the process the normalized and standardized variable is included in the results of the period measurement with all the possible consequences.

5 Of course observations in the form of natural numbers are not ranks but numbers being included in a relative scale. However, such situations happen in statistical and econometric surveys amazingly rarely.

${ }^{6}$ It is worth noticing that the results of the measurement on both variables have subjective character. The lack of a model and subjective approach determine the importance of product durability for a consumer. The variety of graduate and higher level diplomas causes the apparent heterogeneity of the observation results on the variable characterizing a respondent's education.

7 Obviously, the $X_{j}$ variable expresses the importance of the product durability to a respondent.

8 See Churgin (1985), pp. 20-28.

9 The transfer to the measurement in the nominal scale causes the partial loss of information which, on the other hand, increases the analytical potential.

${ }^{10}$ See the works of Wiśniewski (1986), chapter. 4.2 and 6 and Wiśniewski (2009), chapter 6.6.

${ }^{11}$ Keep in mind that the scoring is for the respondent intellectually more challenging than ranking attributes or objects. This is why it requires a little more explanation in the survey questionnaire or by an interviewer.

12 An objective measurement is not possible in this case, because it requires a multidimensional pattern. It is difficult to imagine a situation in which all the pattern components can be specified. You can only define the pattern (the measurand) of a stochastic character, which will make the study object particularly difficult for the respondents to understand.

13 See Wiśniewski (2009), p. 201 and onwards.

${ }^{14}$ Many of measurement results expressed in physical terms have the bottom limit at 0 , e.g. weight, length, volume, etc. 


\section{References}

Churgin, J. (1985). How to Count the Uncountable. Warsaw: Common Knowledge.

Encyclopedia (2004). Kraków: Polish Scientific Publishers PWN, Vol. 10.

Stevens, S.S. (1946). On the Theory of Measurement Scales. Science, Vol. 103, No. 2684.

Wiśniewski, J.W. (1986). Econometric Study of Qualitative Phenomena. Methodological Study, Toruń: Scientific Publishers, Nicolaus Copernicus University.

Wiśniewski, J.W. (2009). Microeconometrics. Toruń: Scientific Publishers, Nicolaus Copernicus University. 